\title{
Regional Changes in Myocardial Strain Predict Ventricular Remodelling after Myocardial Infarction in a Large Animal Model
}

Doyin S. Mansell

University of Bath

Vito D. Bruno

University of Bristol

Eva Sammut

University of Bristol

Amedeo Chiribiri

King's College London

Tom Johnson

University of Bristol

Igor Khaliulin

University of Bristol

Daniel Baz Lopez

University of Bristol

H. S. Gill

University of Bath

Katharine H. Fraser

University of Bath

Michael Murphy

University of Cambridge

Thomas Krieg

University of Cambridge

M. Saadeh Suleiman

University of Bristol

Sarah J. George

University of Bristol

Raimondo Ascione ( $\nabla$ r.ascione@bristol.ac.uk)

University of Bristol

Andrew N. Cookson

University of Bath 


\section{Research Article}

Keywords: Acute myocardial infarction, Cardiac magnetic resonance, Heart failure, Proteomics

Posted Date: May 13th, 2021

DOl: https://doi.org/10.21203/rs.3.rs-479484/v1

License: (c) (i) This work is licensed under a Creative Commons Attribution 4.0 International License. Read Full License 


\section{Regional changes in myocardial strain predict ventricular remodelling after myocardial infarction in a large animal model}

2

D.S. Mansell ${ }^{*}$, V.D. Bruno ${ }^{2 *}$, E. Sammut ${ }^{2}$, A. Chiribiri ${ }^{3}$, T. Johnson ${ }^{2}$, I. Khaliulin ${ }^{2}$, D. Baz Lopez ${ }^{2}$, H.S. Gill ${ }^{1}$, K.H. Fraser ${ }^{1}$, M. Murphy ${ }^{4}$, T. Krieg ${ }^{5}$, M.S. Suleiman ${ }^{2}$, S. George ${ }^{2}$, R. Ascione ${ }^{2 \dagger}$, A.N. Cookson ${ }^{1 \dagger}$

*D.S. Mansell and V.D. Bruno contributed equally and are co-first authors

$\dagger$ A.N. Cookson and R. Ascione contributed equally and are co-senior authors

${ }^{1}$ Department of Mechanical Engineering, University of Bath, Bath, UK

${ }^{2}$ Bristol Heart Institute and Translational Biomedical Research Centre, Faculty of Health Science, University of Bristol, Bristol, UK

${ }^{3}$ School of Biomedical Engineering and Imaging Sciences, King's College London, London, UK

${ }^{4}$ MRC Mitochondrial Biology Unit, University of Cambridge, Cambridge, UK

${ }^{5}$ Department of Medicine, University of Cambridge, Cambridge, UK

\section{Corresponding author:}

Raimondo Ascione, MD, ChM, FRCS,

Bristol Heart Institute, Department of Translational Science,

Bristol Royal Infirmary, level 7, University of Bristol,

Bristol, BS2 8HW, United Kingdom.

Ph: +44 (0) 117 3423286;

E-mail: R.Ascione@bristol.ac.uk

\section{Author's Addresses:}

DS Mansell, HS Gill, KH Fraser and AN Cookson:

Department of Mechanical Engineering,

University of Bath, Bath, BA2 7AY, United Kingdom.

VD Bruno, E Sammut, T Johnson, I Khaliulin, D Baz Lopez, S Suleiman, S George and R Ascione:

Bristol Heart Institute, Department of Translational Science,

Bristol Royal Infirmary, level 7, University of Bristol,

Bristol, BS2 8HW, United Kingdom.

\section{A Chiribiri:}

School of Biomedical Engineering and Imaging Sciences, Westminster Bridge Road, London SE1 7EH, United Kingdom

M Murphy:

MRC Mitochondrial Biology Unit

The Keith Peters Building

Cambridge Biomedical Campus, Hills Road

Cambridge CB2 0XY, United Kingdom

T Krieg:

Department of Medicine,

Addenbrookes Hospital

Box 157

Hills Rd

Cambridge

CB2 0QQ 
56 Article category: Original Article

57 Text Word count: 2998

58 Abstract word count: 196

59 Number of references: 30

60 Number of figures: 6

61 Number of Tables: 2

62 Number of supplemental tables: 4

63

64 


\section{Abstract}

To identify early predictors of late left ventricular remodelling (LVR) post-myocardial infarction (MI) and related molecular signatures, a porcine model of closed-chest balloon MI was used. LVR was assessed by cardiac magnetic resonance imaging (CMRI) at baseline, 12-48 hours (acute), and 5-6 weeks (chronic) post-MI. Changes in myocardial strain and strain rates were derived from CMRI data. Tissue proteomics was compared between infarcted and non-infarcted territories. Peak values of left ventricular (LV) apical circumferential strain (ACS) changed over time together with peak global circumferential strain (GCS) while peak GLS epicardial strains or strain rates did not change over time. LVR post-MI enhanced abundance of 39 proteins in infarcted LV territories, 21 of which correlated with LV equatorial circumferential strain rate (ECSR). The strongest associations were observed for D-3-phosphoglycerate dehydrogenase (D-3PGDH), cysteine and glycine-rich protein-2 (CG-RP-2), and secreted frizzled-related protein 1 (sFRP1). Results indicate that early changes in regional peak ACS predict late LV remodelling and LVR post-MI is associated with augmented levels of D3PGDH and sFRP1, which show the strongest association with peak ECSR. These findings might help to prevent LVR post-MI by influencing/directing LV unloading strategies or by pharmacological control of tissue levels of D-3PGDH and sFRP1.

Keywords: Acute myocardial infarction, Cardiac magnetic resonance, Heart failure, Proteomics. 


\section{Introduction}

Myocardial infarction (MI) leads to left ventricular remodelling (LVR), fibrosis, heart failure (HF), and death ${ }^{1}$ and identifying early predictors of late LVR may benefit patients. Changes in LVEF are not reliable in predicting LVR due to confounding factors and do not represent regional changes ${ }^{2}$. It is suggested that changes in LV myocardial strain (MS) may predict $\mathrm{LVR}^{3}$, tracking contractility both globally and regionally. Hence, quantifying changes in MS early post-MI may predict late LVR. The most effective imaging to measure changes in MS is still debated. Speckle-tracking echocardiography is more common than cardiac magnetic resonance imaging (CMRI ${ }^{4}$, with 2D speckle-tracking echocardiography featuring comparable spatial resolution to CMRI. However, 3D speckle-tracking echocardiography has a lower spatial and temporal resolution. CMRI is considered the reference method for analysis of LV function and mass and provides superior image quality with less interference from anatomical structures ${ }^{5}$, and higher reproducibility, especially for circumferential parameters of strain ${ }^{5,6}$.

The efficacy of MS has been investigated in HF with normal or recovered $\mathrm{LVEF}^{7,8}$; cardiac amyloidosis and hypertrophic cardiomyopathy. A meta-analysis, pooling 16 studies on HF, acute MI and valvular heart disease, has shown global longitudinal strain (GLS) to be a better predictor of mortality than LVEF, with its prognostic ability surpassing that of radial strain or circumferential strain (CS) ${ }^{4,9,10}$. Yet, MS remains largely a research tool. Longitudinal comparisons of MS between healthy and diseased myocardial territories could predict LVR more effectively, but little has been done in this area, partially due to a lack of relevant pre-clinical models ${ }^{11}$. Consequently, the use of GLS as predictor of late LVR post-MI remains controversial ${ }^{12}$. Global strain measures, calculated as averaged values, may result in loss of sensitivity due to missing key information on regional LV areas, a factor also applicable to measurements of LVEF. However, they can reduce the errors that can be associated with regional measures, with the associated improved reproducibility and ease of explaining their popularity ${ }^{5}$. A regional approach to MS, dividing the myocardium into sections and layers (endocardium vs epicardium), has the potential to be a superior predictor of LVR ${ }^{3,4,13-15}$. However, there are no established values for healthy or cardiac disease-specific myocardial strains $9,12,16$

The aim of this study was to identify early strain-based predictors of late LVR post-MI and related molecular signatures in a preclinical model. 
Methods

Ethical Approval for animal procedures

The animal regulated procedures were in line with UK Home Office regulations (Animal Act 1986). The procedures were undertaken under a Project License (No 7008975) granted by the Home Office after formal review and approval by the University of Bristol Animal Welfare and Ethics Review Body (AWERB).

\section{Myocardial infarction model}

Ten Yorkshire female pigs represented the overall cohort of analysis. MI was induced by percutaneous balloon occlusion of the mid portion of either the proximal left anterior descending (LAD, $n=8)$ or the circumflex $(\mathrm{Cx})$ artery $(n=2)$. The coronary occlusion was conducted at the mid portion of the targeted coronary arteries, after the first diagonal or the first obtuse marginal branches. Global and regional morphology, function, volumes and scar size were assessed by CMRI at: a. baseline; b. 12-48 hours (acute); and c. 5-6 weeks (chronic) post-MI. Previous studies have shown that the immune response post MI can be temporally divided into the early proinflammatory phase and the late inflammatory resolution/reparative phase, involving components of both the innate and adaptive immune systems ${ }^{17}$. To better evaluate the remodelling and reparative phase we evaluated the scar 5-6 weeks. Left ventricular remodelling was defined as $10 \%$ or more changes in left ventricular end-systolic and end-diastolic volumes. The additional methods and results for the MI protocol can be found in the Online Supplement.

\section{Deformation analysis}

The strain-based metric used in this study was formulated to be robust and reproducible across sites/users/software, use a transparent, non-proprietary algorithm, and be sufficiently sensitive to characterise local ventricular function in a layer-wise manner, as recently reported by our group ${ }^{18}$. The methodology is summarised on the LV schematic in Figure 1. Slice by slice circumferential strains, $\epsilon$, for the endocardium, were calculated throughout the cardiac cycle for each specimen at each time point, according to Equation 1.

$$
\epsilon=\frac{L-L_{0}}{L_{0}}
$$


The reference length, $\mathrm{L}_{0}$, was the endocardial perimeter length at end-diastole, and this was then compared to the endocardial perimeter length, L, throughout the cardiac cycle for a given CMRI slice. Between seven and nine short-axis slices were contoured depending on the specimen and time point; for example, the degree of eccentric hypertrophy and specimen growth could increase the number of slices for a given specimen over time. Strain rate, $\dot{\epsilon}$, as defined by Equation 2, was also calculated for the circumferential direction, where $L\left(x, t_{n}\right)$ and $L\left(x, t_{n+1}\right)$ are endocardial lengths on one cine MRI stack $(x)$ at consecutive points in time $\left(t_{n}\right), \Delta t$ is the time between successive images, and $L_{0}(x)$ is the end diastolic length for that cine slice.

$$
\dot{\epsilon}=\frac{L\left(x, t_{n+1}\right)-L\left(x, t_{n}\right)}{L_{0}(x) \Delta t}
$$

The same short-axis slices were also divided into three 'vertical regions': apex, equator/mid, and base with two or three slices per region, and regional circumferential strains and strain rates were calculated. Similar analyses were conducted for the epicardium. To assess statistically significant changes through time, peak strain and strain rate values were measured. The strain values were averaged over 2-3 slices per region rather than from only one slice as reported by others ${ }^{19} 2$. Accordingly, the global CS defined here is the average of 7-9 regional slices, rather than of only 3 slices reported by others ${ }^{19}$.

Four-chamber long-axis data were used to investigate LV longitudinal strains and strain rates in both the endocardium and epicardium. Volumes were calculated for LV end-systole and end-diastole by multiplying the area within the endocardial contour for a given slice by the slice thickness, and then all slice volumes were summed to give the ventricle blood pool volume. LV end-systolic volume indexed to weight (LVESVi) was calculated by dividing the end-systolic volume by the body surface area (BSA) of the animal. BSA was found through the relation suggested by Kelley et al. shown in Equation $3^{20}$.

$$
B S A\left(m^{2}\right)=0.0734 * \text { Weight }(k g)^{0.656}
$$

The endocardial and epicardial contours were manually traced on all short and long axis images in the commercially available software package OsiriX (Pixmeo, Geneva, Switzerland), by one experienced user, and checked by another. Contours were analysed and strain \& strain rate values were calculated using an in-house MATLAB script (Release 2017b, The MathWorks, Inc., Natick, Massachusetts, United States). 
Proteomics analysis of infarcted vs remote viable myocardium was performed in five animals in keeping with established methods ${ }^{21}$. Tissue homogenization was obtained with ceramic beads with Ripa Buffer and a protease and phosphatase inhibitors cocktail. The BCA method was used to quantify the protein concentration and samples were prepared at $2 \mathrm{mg} / \mathrm{ml}$ for the mass spectroscopic analysis (MSA). Additional details are available in the Online Supplement.

\section{Statistical Analysis}

Non-parametric analysis was performed. Variables are presented as medians and confidence intervals. LVEF, peak values of global LS, global and regional CS, and peak values of corresponding strain rates measured at acute and chronic time-points post-MI were compared with the baseline data using a Kruskall-Wallis test. Observed significant differences were analysed further by using Mann-Whitney U tests. A p-value of $<0.05$ was considered statistically significant, but due to the high number of hypotheses tested, Bonferroni corrections were performed which suggested a $\mathrm{p}<0.0024$ as statistical significance. One-way ANOVA was used for initial assessment with Gabriel's test to find differences between pairs of means. Linear regression and correlation analyses were performed to assess relationships between the scar weight and other mechanical properties, and between biomarker expression and strain. Statistical analyses were performed in IBM SPSS (IBM Corp. Released 2015).

\section{Proteomics data analysis}

For each protein, an abundance ratio between infarcted and non-infarcted samples was calculated. Proteins found to be at least two-fold more expressed in the infarcted myocardium were correlated with the endocardial strain data of the acute phase, with those showing the strongest correlation $\left(R^{2} \geq 0.95\right)$ being more closely evaluated; a series of univariate linear regression models were performed to correlate each identified protein to each mechanical variable. This statistical analysis was conducted using R version 3.4.4. For western blotting analysis quantification of band intensity was performed using AlphaEase v5.5 software followed by background subtraction and correction for protein loading. For evaluation of the differences between the protein expression in the non-ischaemic and ischaemic myocardium, the two-tailed unpaired Student's t-test was used. 


\section{Results}

Animals were 5-6 months old. Weight range was $55-70 \mathrm{~kg}$, median $62.5 \mathrm{~kg}$ at the time of MI and $72-92 \mathrm{~kg}$, median $84 \mathrm{~kg}$ at termination.

\section{Characterisation of MI by CMRI and serial troponin I release}

CMRI outcome is shown in Table S1, Online Supplement. Overall mean LVEF dropped from 56.6\% $\pm 2.5 \%$ at baseline to $45.3 \% \pm 7.6 \%$ at 4 to 72 hours (acute) and to $49 \% \pm 4.6 \%$ at 5-6 weeks (chronic). Mean LV scar size was $16.9 \mathrm{~g} \pm 9.1 \mathrm{~g}$ at the acute time point and $9.38 \mathrm{~g} \pm 5.62 \mathrm{~g}$ at the chronic time-point. Mean LV end-diastolic volume (LVEDV) increased from $131 \pm 11.3 \mathrm{~mL}$ at baseline to $144.3 \pm 5 \mathrm{~mL}$ at $12-48$ hours and to $194.6 \pm 27.6 \mathrm{~mL}$ at 5-6 weeks post-MI suggesting occurrence of significant LVR over time. Mean LV end-systolic volume (LVESV) increased from $56.8 \pm 4.9 \mathrm{~mL}$ at baseline to $77.8 \pm 16.4 \mathrm{~mL}$ at $12-48$ hours and to $100.4 \pm 17.7 \mathrm{~mL}$ at $4-6$ weeks. Representative longitudinal CMRI scans from the same experiment are reported in Figure 2 A-C and in Supplemental Videos 1-3). The peak of troponin I release was recorded at 4 hours with a value of $49.6 \pm 39.71$ ng/ml (Table S2, Online Supplement).

\section{Myocardial strains}

Myocardial strains were calculated from all 10 MI experiments ( $n=8$ LAD territory and $n=2$ CX territory; see Figure S1 in Supplemental file). The occurrence of MI in these two different coronary territories caused tissue damage in the apical and lateral LV wall regions respectively. For the majority of the analysis focusing on LV global metrics, such as LVEF, GCS and GLS, myocardial strains from all 10 experiments were included as these indices should be able to characterise the severity of an infarct \& the subsequent LV remodelling regardless of the affected coronary territory. LV remodelling, occurring due to myocardial tissue's response to the imposed occlusion, encompasses changes in ventricular shape, volume, and function throughout the cardiac cycle. Similarly, MRI data from all baseline, pre-MI scans were retained in the analysis. Nevertheless, for, the statistical analysis of myocardial strain changes in ACS at acute and chronic timepoints only data from the 8 LAD experiments were included, as only these cases were expected to determine an MI affecting the apical region i.e. that covered by the ACS metric. The same approach was taken for the other regional strains ECS and BCS. An evaluation of the regional strains for the CX territory was not performed, because with only $n=2$ experiments in this sub-group such an evaluation would not have been meaningful. 
Changes in myocardial strains over time for all animals are shown in Table $\mathbf{1}$ and Table S3 (Online

Supplement). Assessment of endocardial and epicardial GLS did not differ at the acute or chronic time-points versus baseline strain. Endocardial and epicardial strain rates did not differ at the acute or chronic time-point compared to baseline values. No correlations were observed between scar weight and GLS or strain rate.

\section{Short-axis global and regional LV strains}

Endocardial global CS (GCS) and apical CS (ACS) are shown in Figure 3 A-D. Endocardial ACS decreased significantly at the chronic time point $(-19.1 \%, \mathrm{p}=0.002)$ point compared to baseline $(-37.5 \%)$, whereas the change at the acute time point did not reach statistical significance $(-17.7 \%, \mathrm{p}=0.004)$ (Table 1). GCS showed a significant change at the acute time-point $(-23.8 \%, \mathrm{p}=0.002)$ vs. baseline $(-34.9 \%)$, whereas the change observed at the chronic time-point did not reach statistical significance $(-27.7 \%, \mathrm{p}=0.006)$. The endocardial equatorial CS (ECS) and basal CS (BCS) strains showed no statistical changes across the two time-points, with all p>0.01 (Table S4, Online Supplement). No significant changes were seen in epicardial CS, with all p>0.03 (Table S4). Endocardial and epicardial strain rates did not differ at the acute or chronic time-points vs. baseline ( $p>0.008$;

Figure 3 E-G and Table S4). No significant correlations were observed between scar weight and circumferential strains. The intra-observer variability for LVEF and LVEDV were $3 \%$ and $2 \%$ respectively, and the inter-observer variation were $8 \%$ and $7 \%$ respectively.

\section{Global Function and Associations with Strain}

LVEF and left ventricular end systolic volume index (LVESVi) were measured by established CMRI methods (Table S1, Online Supplement) with relevant strain contours derived by hand (Table 1). LVEF dropped significantly only at the acute time-point vs. baseline ( $\mathrm{p}=0.0023)$ (Table 1 and Figure 3 E-G). Scatterplots of GCS versus LVEF $(R=-0.95, p<0.0001)$ and GLS versus LVEF $(R=-0.73, p=0.0006)$ indicated strong correlations (Figure 4). LV volumes indexed to weight were assessed given the substantial weight gain observed from acute to chronic time-points. This showed that there were no significant changes in LVESVi from acute to chronic time-points (Table 1). No difference was seen in LVEF values between those measured by CMRI and the relevant strain contours derived by hand.

Myocardial proteomics, LV strains, and validation of D-3PGDH and sFRP1 by western blotting

Proteomics data and correlation with LV strains are reported in Figure 5 and Table 2. 5981 proteins were identified, and 39 proteins were increased in infarcted territories (Figure 5). For the analysis correlating 
proteomics with strains, proteomics data from 4 hearts was used as strains were not available for the 5 experiments. Significant linear correlations were found between endocardial circumferential strain rate (ECSR) and 21 of the proteins increased in the infarcted territories (Table 2). The proteins showing the strongest correlation $\left(R^{2} \geq 0.95\right)$ with the ECSR were: D-3-phosphoglycerate dehydrogenase $\left(\mathrm{D}-3 \mathrm{PGDH}, \mathrm{R}^{2}=0.96, \mathrm{p}=\right.$ $0.01)$, cysteine and glycine-rich protein-2 $\left(\mathrm{CG}-\mathrm{RP}, \mathrm{R}^{2}=0.95, \mathrm{p}=0.02\right)$, and secreted frizzled-related protein 1 (sFRP1, R ${ }^{2}$ 0.96, $\mathrm{p}=0.01$ ). Western blotting for D-3PGDH and sFRP1 confirmed that the level of D-3PGDH and sFRP1 protein in the infarcted myocardium was significantly increased compared to the non-infarcted myocardium (both $\mathrm{P}<0.05$, Figure 6, Figure S2 in Supplemental file). Western blotting for CG-RP showed no difference.

\section{Discussion}

This study identifies an association between early change in regional strain, late LVR post-MI and enhanced abundance of D-3PGDH and sFRP1 proteins. An early change in regional ACS was observed (but not in global measures such as GCS, LVEF or GLS), which was predictive of late LVR. Additionally, LVR post-MI is associated with an hyperexpression of 39 myocardial proteins, of which 21 correlated specifically with ECSR, with D-3PGDH and sFRP1 exhibiting the strongest correlation with ECSR.

Our findings show early changes in regional ACS and GCS strains reflecting accurately the affected myocardial territories. This was associated with early changes in strain that predicted late LVR: ACS: baseline: $-37.5 \%$, chronic: $-19.1 \%, \mathrm{p}=0.002$; GCS: baseline: $-34.9 \%$, acute: $-23.8 \%, \mathrm{p}=0.002$, chronic: $-27.7 \%, \mathrm{p}=0.006$.

GLS has been suggested as a predictor of late LVR in STEMI patients ${ }^{10}$ and in an open-chest coronary ligation porcine MI model ${ }^{11}$. It is argued that GLS may predict LVR as the LV apical region affected by ischemia contains more longitudinal fibres, which contributes more to the local contractile performance and are less affected by ischemia ${ }^{12}$. However, the distribution of the circumferential fibres across the LV might reflect changes to longitudinal and circumferential deformations ${ }^{12}$, therefore suggesting that CS metric might add significantly to gauge predictive information on myocardial deformation ${ }^{22}$.

In our study GLS did not change over time. The results seem to suggest that regional CS might be more sensitive than GLS in predicting late LVR and quantifying LV function. Averaging strains over few slices within a specific LV region, as opposed to inspecting individual slices or global metrics, might ensure that small differences in image location (from patient movement or from scans at different times or different patients), become less critical when comparing data, boosting the reproducibility and robustness of the method. In 
addition, performing strain over smaller volume/regions is associated with less variation, hence with higher potential of identifying smaller changes. Accordingly, CS has been shown to be an effective indicator of MI, marker of LV function, and infarct transmurality (25). These findings, if confirmed, might affect the type and timing of pharmacological and/or mechanical LV unloading approaches post-MI to prevent heart failure (27).

Correlations were also found between GCS and LVEF as well as between GLS and LVEF. Both GCS and LVEF were calculated using the same short-axis data, and so a strong correlation was expected based on geometrical considerations. Long-axis data was used for GLS, so the correlation found between these parameters suggests that GLS might be able to detect MI and changes to LV function in keeping with findings by others ${ }^{26}$.

The occurrence of MI and related ischemia/reperfusion injury trigger a storm of molecular signalling, cellular remodelling, inflammatory reaction and fibrosis leading to scar formation and LVR ${ }^{27,28}$. Farah and colleagues ${ }^{29}$ defined LV remodelling as an increase of $10 \%$ in ventricular end-systolic or end-diastolic diameter, and found a $58 \%$ incidence of LV remodelling after an anterior MI compared with other studies. In the Acute Myocardial Infarction Contrast Imaging (AMICI) trial, the term 'reverse REM' was employed to denote a >10 \% reduction in LVESV found at 6 months in $39 \%$ of patients following $\mathrm{PPCI}^{30}$, being the only independent predictor of 2 year event-free survival. Based on this definition we found that $75 \%$ of our experiments had an LV remodeling at both LVEDV and LVESV at 5-6 weeks at serial CMR.

Binek et al. found that ischaemia triggers changes in the levels of many myocardial proteins, some of which are linked to contractile function or systolic wall thickness ${ }^{31}$. Proteomics analysis in this study showed 39 hyper expressed proteins, 21 of these being strongly correlated with early changes in regional ECSR. The western blotting analysis showed that D-3PGDH and sFRP1 are significantly expressed within the infarcted myocardium. This finding might indicate their involvement in the early changes in ECSR as well as in determining LVR post-MI. D-3PGDH is the key enzyme for the L-Serine biosynthesis pathway that branches from glycolysis. It participates in a metabolic network interlinking folate and methionine cycles to support cell proliferation and an amplification of function has been associated with a pro-oncogenic role ${ }^{32}$. sFRP1 acts as an inhibitor of the Wnt signalling pathway by binding to Wnt proteins and preventing their association with Frizzled receptors (34). Interestingly, sFRP1 protein has been associated with reduced scar size, improved cardiac function and decreased neutrophil infiltration in a mice model of coronary ligation, indicating a protective role of this protein via reduction of post-MI inflammation ${ }^{34}$. This anti-inflammatory role has been 
suggested by others in rodents but not in pigs. SFRP1 to suppress the Wnt pathway has potential clinical translation for novel therapies aiming to reduce scar size post-MI and warrants further investigation.

There are limitations to this study. The animals did not have atherosclerotic disease, which might have determined a different proteomic profile. However, the MI size and other CMRI measures were in keeping with what observed in humans. In addition, the animals gained a substantial amount of weight over the study period with a possible confounding effect on scar size and proteomics. However, it has been suggested that the use of CMRI parameters indexed to the weight of the animal can minimise this effect. Finally, a relatively small number of animals $(n=10)$ was used, with strain analyses and proteomics undertaken on sub-groups: nonparametric statistical tests were used to compensate. Another limitation is related to the lack of information on the dynamic changes that occur after MI: due to the nature of our study design we were unable to characterise the dynamic proteomic processes as previously described by other authors ${ }^{31}$.

In conclusion, this study reveals novel associations between MI, early changes in regional ACS, prediction of late LV remodelling, the related abundance of myocardial D-3PGDH and sFRP1, and their association with ECSR. These findings might have clinical implications: the observation of early changes in regional strain may influence type and timing of pharmacological and/or mechanical LV unloading to prevent LV remodelling; in addition, future therapies modulating the tissue levels of D-3PGDH or delivering sFRP1might help reducing LV remodelling post-MI. 


\section{References}

1. Benjamin, E. J. et al. Heart disease and stroke statistics - 2018 update: A report from the American Heart Association. Circulation 137, E67-E492 (2018).

2. Cameli, M. et al. Echocardiographic assessment of left ventricular systolic function: from ejection fraction to torsion. Heart Fail. Rev. 21, 77-94 (2016).

3. Altiok, E. et al. Layer-specific analysis of myocardial deformation for assessment of infarct transmurality: comparison of strain-encoded cardiovascular magnetic resonance with 2D speckle tracking echocardiography. Eur. Hear. J. - Cardiovasc. Imaging 14, 570-578 (2013).

4. Chan, J. et al. Differentiation of Subendocardial and Transmural Infarction Using TwoDimensional Strain Rate Imaging to Assess Short-Axis and Long-Axis Myocardial Function. J. Am. Coll. Cardiol. 48, 2026-2033 (2006).

5. Pedrizzetti, G., Claus, P., Kilner, P. J. \& Nagel, E. Principles of cardiovascular magnetic resonance feature tracking and echocardiographic speckle tracking for informed clinical use. $J$. Cardiovasc. Magn. Reson. 18, 51 (2016).

6. Voigt, J. U. et al. Strain-rate imaging during dobutamine stress echocardiography provides objective evidence of inducible ischemia. Circulation 107, 2120-2126 (2003).

7. Adamo, L. et al. Abnormal Global Longitudinal Strain Predicts Future Deterioration of Left Ventricular Function in Heart Failure Patients With a Recovered Left Ventricular Ejection FractionCLINICAL PERSPECTIVE. Circ. Hear. Fail. 10, e003788 (2017).

8. Kraigher-Krainer, E. et al. Impaired Systolic Function by Strain Imaging in Heart Failure With Preserved Ejection Fraction. J. Am. Coll. Cardiol. 63, 447-456 (2014).

9. Ersbøll, M. et al. Prediction of All-Cause Mortality and Heart Failure Admissions From Global Left Ventricular Longitudinal Strain in Patients With Acute Myocardial Infarction and Preserved Left Ventricular Ejection Fraction. J. Am. Coll. Cardiol. 61, 2365-2373 (2013).

10. Gavara, J. et al. Prognostic Value of Strain by Tissue Tracking Cardiac Magnetic Resonance After ST-Segment Elevation Myocardial Infarction. JACC Cardiovasc. Imaging (2017) doi:10.1016/J.JCMG.2017.09.017.

11. Howard-Quijano, K. et al. Left ventricular endocardial and epicardial strain changes with 
apical myocardial ischemia in an open-chest porcine model. Physiol Rep 4, (2016).

12. Rademakers, F. \& Nagel, E. Is Global Longitudinal Strain a Superior Parameter for Predicting Outcome After Myocardial Infarction? JACC: Cardiovascular Imaging vol. 11 1458-1460 (2018).

13. Opdahl, A., Helle-Valle, T., Skulstad, H. \& Smiseth, O. A. Strain, Strain Rate, Torsion, and Twist: Echocardiographic Evaluation. Curr. Cardiol. Rep. 17, 15 (2015).

14. Koos, R. et al. Layer-specific strain-encoded MRI for the evaluation of left ventricular function and infarct transmurality in patients with chronic coronary artery disease. Int. J. Cardiol. 166, 85-89 (2013).

15. Leitman, M. et al. Circumferential and Longitudinal Strain in 3 Myocardial Layers in Normal Subjects and in Patients with Regional Left Ventricular Dysfunction. J. Am. Soc. Echocardiogr. 23, 64-70 (2010).

16. Smiseth, O. A., Torp, H., Opdahl, A., Haugaa, K. H. \& Urheim, S. Myocardial strain imaging: how useful is it in clinical decision making? Eur. Heart J. 37, 1196-207 (2016).

17. Lai, S. L., Marín-Juez, R. \& Stainier, D. Y. R. Immune responses in cardiac repair and regeneration: a comparative point of view. Cell. Mol. Life Sci. 76, 1365-1380 (2019).

18. Mansell, D. S. et al. Comparison of the within-reader and inter-vendor agreement of left ventricular circumferential strains and volume indices derived from cardiovascular magnetic resonance imaging. PLoS One 15, 1-16 (2020).

19. Bachner-Hinenzon, N. et al. Strain Analysis in the Detection of Myocardial Infarction at the Acute and Chronic Stages. Echocardiography 33, 450-458 (2016).

20. Kelley, K. W., Curtis, S. E., Marzan, G. T., Karara, H. M. \& Anderson, C. R. Body Surface Area of Female Swine. J. Anim. Sci. 36, 927-930 (1973).

21. Barallobre-Barreiro, J. et al. Proteomics Analysis of Cardiac Extracellular Matrix Remodeling in a Porcine Model of Ischemia/Reperfusion Injury. Circulation 125, 789-802 (2012).

22. Chen, R., Zhu, M., Sahn, D. J. \& Ashraf, M. Non-invasive evaluation of heart function with four-dimensional echocardiography. PLoS One 11, e0154996 (2016).

23. Bachner-Hinenzon, N. et al. Strain Analysis in the Detection of Myocardial Infarction at the 
Acute and Chronic Stages. Echocardiography 33, 450-458 (2016).

24. Uriel, N., Sayer, G., Annamalai, S., Kapur, N. K. \& Burkhoff, D. Mechanical Unloading in Heart Failure. J. Am. Coll. Cardiol. 72, 569-580 (2018).

25. Birks, E. J. Molecular Changes After Left Ventricular Assist Device Support for Heart Failure. Circ. Res. 113, 777-791 (2013).

26. Fent, G. J. et al. The utility of global longitudinal strain in the identification of prior myocardial infarction in patients with preserved left ventricular ejection fraction. Int. J. Cardiovasc. Imaging 33, 1561-1569 (2017).

27. Frangogiannis, N. G. The immune system and cardiac repair. Pharmacol. Res. 58, 88-111 (2008).

28. de Boer, R. A. et al. Towards better definition, quantification and treatment of fibrosis in heart failure. A scientific roadmap by the Committee of Translational Research of the Heart Failure Association (HFA) of the European Society of Cardiology. Eur. J. Heart Fail. 21, 272-285 (2019).

29. Farah, E. et al. Prevalence and predictors of ventricular remodeling after anterior myocardial infarction in the era of modern medical therapy. Med. Sci. Monit. 18, 276-281 (2012).

30. Funaro, S. et al. Incidence, determinants, and prognostic value of reverse left ventricular remodelling after primary percutaneous coronary intervention: Results of the Acute Myocardial Infarction Contrast Imaging (AMICI) multicenter study. Eur. Heart J. 30, 566575 (2009).

31. Binek, A. et al. Proteomic footprint of myocardial ischemia/reperfusion injury: Longitudinal study of the at-risk and remote regions in the pig model. Sci. Rep. 7, 1-16 (2017).

32. Locasale, J. W. et al. Phosphoglycerate dehydrogenase diverts glycolytic flux and contributes to oncogenesis. Nat. Genet. 43, 869-74 (2011).

33. Pereira, C., Schaer, D. J., Bachli, E. B., Kurrer, M. O. \& Schoedon, G. Wnt5A/CaMKII Signaling Contributes to the Inflammatory Response of Macrophages and Is a Target for the Antiinflammatory Action of Activated Protein C and Interleukin-10. Arterioscler. Thromb. Vasc. Biol. 28, 504-510 (2008). 
34. Dufourcq, P. et al. FrzA, a Secreted Frizzled Related Protein, Induced Angiogenic Response.

404 Circulation 106, 3097-3103 (2002).

405 


\section{Acknowledgements}

The authors would like to thank the staff at the University of Bristol Translational Biomedical Research Centre, a national research facility for large animal co-funded by the British Heart Foundation and the Medical Research Council.

\section{Authors Contribution}

DSM: design of the study, computerised model after CMR, strain measurement and statistical analysis, drafting the report, preparation of figures. VDB: design of the study, conducting the MI model, collecting myocardial sampling for proteomics analysis, Statistical analysis for proteomics data, drafting the report, preparation of figures ES: design of the study, conducting MI model, CMR acquisition, interpretation and protocol preparation of figures. AC: supervisor for CMR and data interpretation TJ: design of the study, conduction of MI model IK: Western Blot analysis and preparation of the relative picture. DBL: blood sampling collection, preparation and analysis, tissue sample collection, preparation and analysis, proteomics sampling. HS GILL: conceptualisation, study design, computerised model of CMR KH Fraser: conceptualisation, study design, computerised model of CMR MM: design of the study TK: design of the study MS: design of the study SG: design of the study RA: secured funding, conceptualisation, design of the study, protocol development, conducting the model, drafting and revision of manuscript, final revision of the submitted paper, senior supervisor of the project, corresponding author. ANC: conceptualisation, design of the study, protocol development, revision of manuscript, final supervision of the submitted paper, senior supervisor of the project. All the authors read the manuscript.

\section{Conflict of interest}

The authors declare that there are no competing risk interest in this work. 
428 Additional Information:

\section{$429 \quad$ Funding}

430 This work was supported by a Great West 4 (GW4) Initiator grant to Ascione (GW4-AF4-011) and partially by the

431 University of Bath. In addition, this work was supported by the following grants to Ascione: Medical Research

432 Council (MRC): MR/L012723/1; British Heart Foundation (BHF): IG/14/2/30991, PG/10/40/28369,

433 PG/07/046/22772, and PG/16/104/32652), and by a Developmental Pathway Funding Scheme (DPFS) grant to

434 Murphy, Krieg and Ascione (MR/M015769/1). Mansell was supported by an EPSRC DTP.

435

436 Ethics

437 The procedures were undertaken at the University of Bristol Translational Biomedical Research Centre in accordance 438 with the United Kingdom Animal (Scientific Procedures) Act, 1986 (Home Office Project Licence No 7008975) and the 439 European Union Directive 2010/63/EU. Female Yorkshire pigs ( $\mathrm{n}=10$; weight $62.3 \mathrm{~kg} \pm 5.55 \mathrm{~kg}$ ) were used in the study. 440 Regulated procedures were in line with Home Office (Animal Act 1986) as described in approved PPL 7008975.

441 The study was carried out in compliance with the ARRIVE guidelines.

443 Patient and Public Involvement

444 This research was done without patient involvement. Patients were not invited to comment on the study design and 445 were not consulted to develop patient relevant outcomes or interpret the results. Patients were not invited to contribute 446 to the writing or editing of this document for readability or accuracy. 
Figures Legends

\section{Figure 1: Depiction of workflow from imaging to derived final strains.}

Short-axis CMRIs with endocardial (green) and epicardial (blue) borders were traced, stacked and grouped into three regions: base, equator/mid, and apex. Strain was calculated for all slices, and the mean was then found by averaging strains in their regions. Finally, the resultant regional and global circumferential strains were found for each time point.

\section{Figure 2: Representative longitudinal CMRI imaging of failing Left Ventricle}

From left to right showing CMRI images from the same animal at baseline (A), acute (B) and chronic (C) time-points. Large images show stills of cinematic imaging in 3-chamber view orientation at end diastole demonstrating progressive thinning of the mid to apical antero-septal wall (orange arrows). Inset images show corresponding late gadolinium enhancement imaging demonstrating full thickness late gadolinium enhancement (red arrows).

\section{Figure 3}

\section{A-D: Overtime changes in apical and global circumferential strains.}

$\mathrm{A}=$ Endocardial apical circumferential strain (ACS); B = Epicardial apical circumferential strain $(\mathrm{ACS}) ; \mathrm{C}=$ Endocardial global circumferential strain (GCS); D = Epicardial global circumferential strain (GCS). The two data points at the chronic time point with a red cross (in endocardial ACS and GCS) represent outliers greater than the third quartile plus 1.5 times the interquartile range.

\section{E-G: Overtime changes in LVEF, endocardial and epicardial ACS rates.}

$\mathrm{E}=$ Left Ventricular Ejection Fraction $(\mathrm{LVEF}) ; \mathrm{F}=$ Endocardial apical circumferential strain (ACS) rates;

$\mathrm{G}=$ Epicardial ACS rates. The asterisk denotes changes considered significant with $\mathrm{p}<0.0024$. Statistical test: Mann-Whitney $(*=$ identifies significant difference)"

\section{Figure 4: Correlation between endocardial GCS and GLS with LVEF}

Scatterplot between endocardial GCS and LVEF (A) and between endocardial GLS and LVEF (B). Each individual porcine specimen is denoted by a different marker and three different line colours are used to indicate the experimental time point of baseline, acute, and chronic.

\section{Figure 5: Volcano plot representation of proteomics}

Abundance ratios for changes in each protein are shown as $\log 10$ of p-value of infarcted/health segments within the same hearts $(n=5)$ 
479 Figure 6: Quantification of D-3PGDH and sFRP1 proteins by western blotting

480 All data presented as Mean \pm SEM; n=5 in each group. Quantification of D-3-phosphoglycerate dehydrogenase (D-3PGDH) and 481 secreted frizzled-related protein 1 (sFRP1) in lysates of the infarcted myocardium (I) and non-infarcted myocardium (N). $482 \mathbf{A}=$ Representative western blot of D-3PGDH; $\mathbf{B}=$ Densitometric quantification of D-3PGDH; $\mathbf{C}=$ Representative western blot for 483 sFRP1; D=Densitometric quantification of sFRP1 expression; $\mathbf{E}=$ Representative western blot for anti-glyceraldehyde 3-phosphate 484 dehydrogenase (GAPDH, used for control of protein loading); $* \mathrm{P}<0.05$ vs. non-ischaemic myocardium. MW: molecular weight. 485 Statistical Test used: Mann-Whitney. (Figure S1 shows the full blots for all the proteins shown in this figure) 
Table 1: Changes in myocardial strains over time.

\begin{tabular}{|c|c|c|c|c|c|c|c|c|c|c|c|}
\hline \multirow[t]{2}{*}{ Parameter } & \multirow[t]{2}{*}{ Baseline } & \multicolumn{2}{|c|}{$95 \% \mathrm{CI}$} & \multirow{2}{*}{$\begin{array}{l}\text { Acute } \\
\text { MI }\end{array}$} & \multicolumn{2}{|c|}{$95 \% \mathrm{CI}$} & \multirow[t]{2}{*}{ p-value } & \multirow{2}{*}{$\begin{array}{c}\text { Chronic } \\
\text { MI }\end{array}$} & \multicolumn{2}{|c|}{$95 \% \mathrm{CI}$} & \multirow[t]{2}{*}{ p-value } \\
\hline & & Upper & Lower & & Upper & Lower & & & Upper & Lower & \\
\hline $\begin{array}{c}\text { Endocardial } \\
\text { ACS }(\%)\end{array}$ & -37.5 & -31.2 & -49.4 & -17.7 & -16.8 & -27.0 & 0.004 & -19.1 & -12.3 & -26.3 & $0.002 *$ \\
\hline $\begin{array}{c}\text { Endocardial } \\
\text { GCS }(\%)\end{array}$ & -34.9 & -30.5 & -28.4 & -23.8 & -21.1 & -26.1 & $0.002 *$ & -27.7 & -17.8 & -31.6 & 0.006 \\
\hline $\begin{array}{c}\text { Endocardial } \\
\text { GLS }(\%)\end{array}$ & -24.7 & -19.9 & -28.9 & -18.0 & -14.8 & -22.7 & 0.008 & -21.7 & -12.8 & -27.6 & 0.171 \\
\hline LVEF (\%) & 57 & 52 & 62 & 45 & 40 & 50 & $0.002 *$ & 50 & 46 & 54 & 0.019 \\
\hline $\begin{array}{c}\text { LVESVi } \\
\left(\mathrm{ml} / \mathrm{m}^{2}\right)\end{array}$ & 35.9 & 26.8 & 49.1 & 52.1 & 45.3 & 60.3 & 0.045 & 50.0 & 37.4 & 63.3 & 0.127 \\
\hline
\end{tabular}

488

489

$490 C I=$ Confidence interval; $A C S=$ Apical circumferential strain; $G C S=$ Global circumferential

491 strain; GLS=Global longitudinal strain; LVESVi=Left ventricular end-systolic volume index;

$492 \quad P$-values are for differences compared to baseline results. * denotes changes significant to $p$

493

$<0.0024$.

494

495 
Table 2: Correlation between endocardial ECSR and most overexpressed proteins after MI

\begin{tabular}{|c|c|c|c|}
\hline Protein & Infarcted vs Healthy & \multicolumn{2}{|c|}{ Correlation with ECSR } \\
\hline Accession & Fold Change & R-squared & p-value \\
\hline A5GFY8 & 2.027 & 0.96 & 0.01 \\
\hline I3LB66 & 2.39 & 0.96 & 0.01 \\
\hline F1RYJ8 & 2.42 & 0.95 & 0.02 \\
\hline F1RVS9 & 2.65 & 0.94 & 0.02 \\
\hline F1SCR9 & 2.09 & 0.94 & 0.02 \\
\hline Q6EEI0 & 2.66 & 0.94 & 0.02 \\
\hline F1RF27 & 3.63 & 0.93 & 0.03 \\
\hline F1RF28 & 2.36 & 0.93 & 0.03 \\
\hline F1S5Q1 & 2.6 & 0.93 & 0.03 \\
\hline I3L7W9 & 2.01 & 0.93 & 0.03 \\
\hline F1RPQ0 & 2.15 & 0.92 & 0.03 \\
\hline F1SLT8 & 2.19 & 0.92 & 0.03 \\
\hline F1SSF7 & 2.52 & 0.92 & 0.03 \\
\hline F1S1D2 & 2.81 & 0.91 & 0.04 \\
\hline F1S6B5 & 4.02 & 0.91 & 0.04 \\
\hline F1SJL4 & 2.26 & 0.91 & 0.04 \\
\hline Q29116 & 2.9 & 0.91 & 0.04 \\
\hline F1RQI0 & 5.29 & 0.9 & 0.04 \\
\hline F1RQI2 & 12.1 & 0.9 & 0.04 \\
\hline F1RIP3 & 2.29 & 0.9 & 0.05 \\
\hline I3LPW3 & 2.63 & 0.89 & 0.05 \\
\hline B3F0B7 & 2.13 & 0.86 & 0.06 \\
\hline
\end{tabular}




\section{Figures}

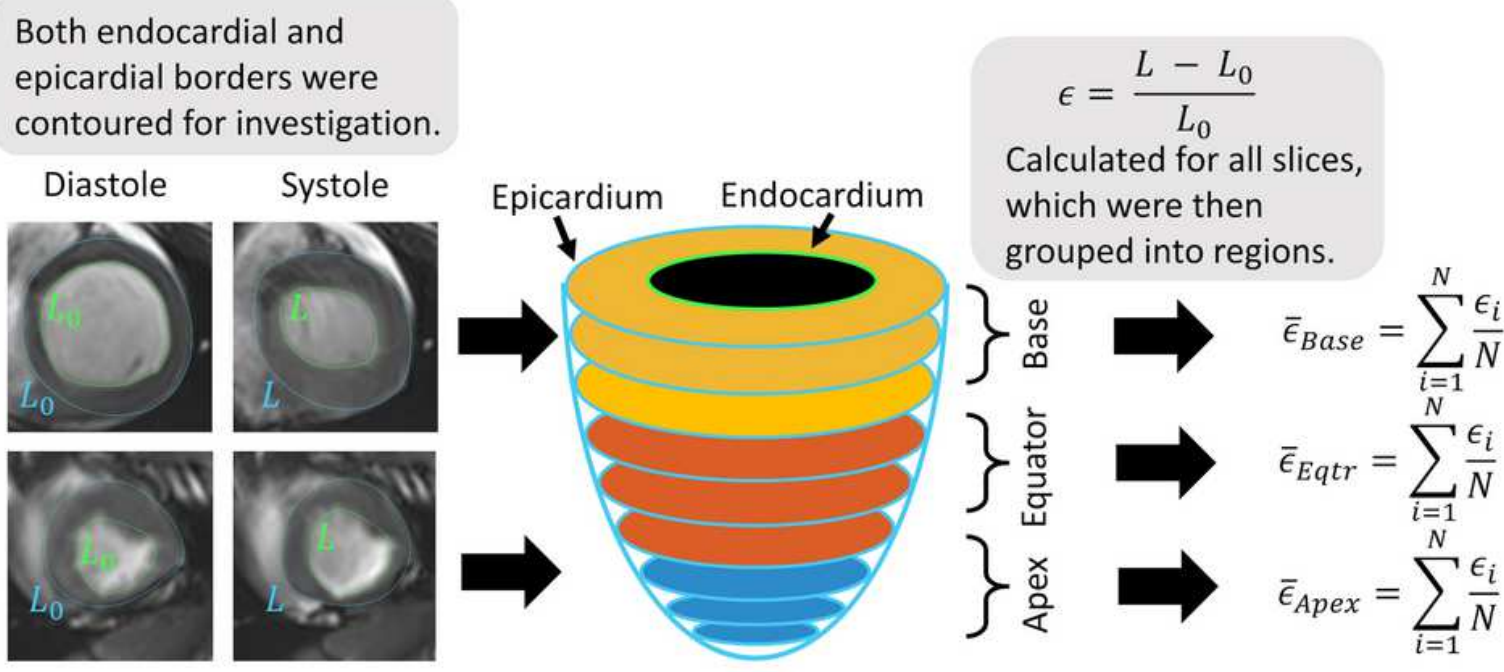

Both endocardial and epicardial borders were contoured for investigation.
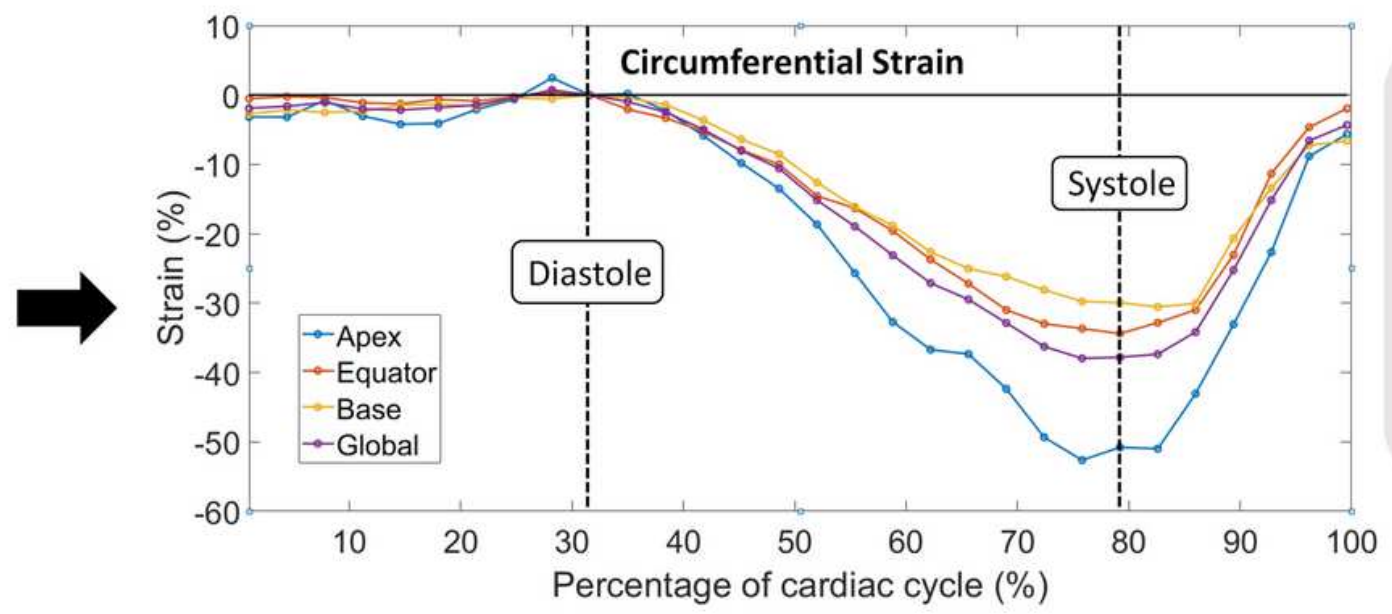

$\mathrm{N}$ is the number of slices in a region.

This was either 2 or 3 depending on the MRI slices acquired.
Regional and global circumferential strains were calculated from short-axis data. Only global longitudinal strain was calculated from the long-axis data.

\section{Figure 1}

Depiction of workflow from imaging to derived final strains. Short-axis CMRIs with endocardial (green) and epicardial (blue) borders were traced, stacked and grouped into three regions: base, equator/mid, and apex. Strain was calculated for all slices, and the mean was then found by averaging strains in their regions. Finally, the resultant regional and global circumferential strains were found for each time point. 


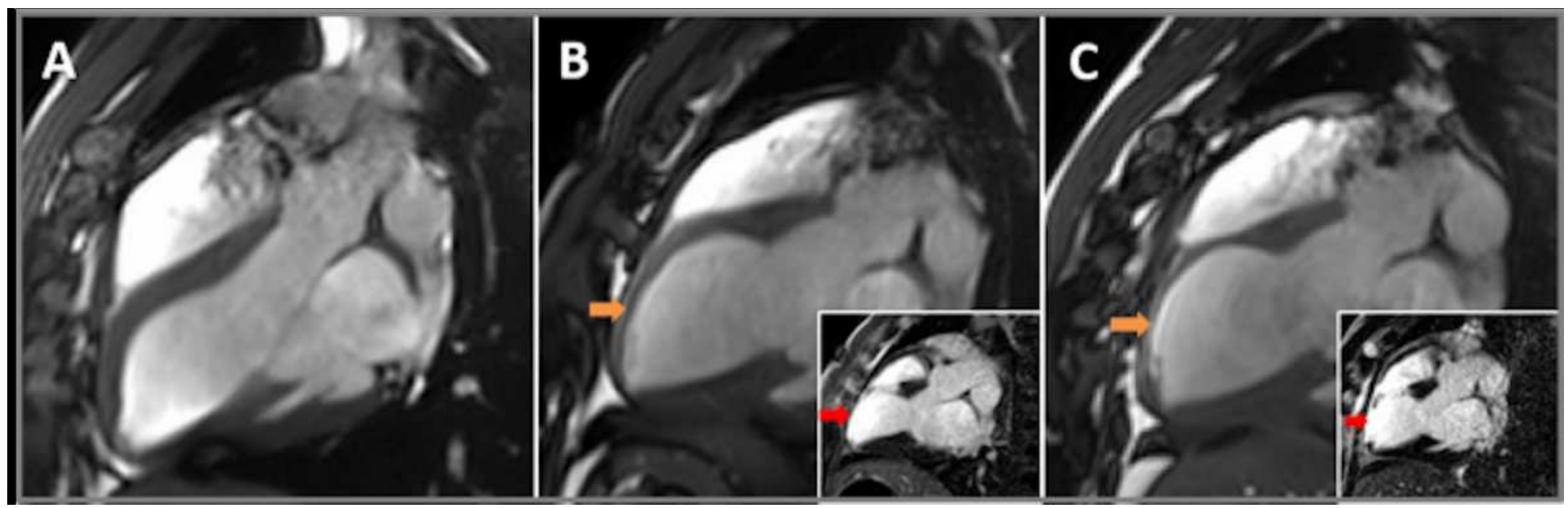

Figure 2

Representative longitudinal CMRI imaging of failing Left Ventricle From left to right showing CMRI images from the same animal at baseline (A), acute (B) and chronic (C) time-points. Large images show stills of cinematic imaging in 3-chamber view orientation at end diastole demonstrating progressive thinning of the mid to apical antero-septal wall (orange arrows). Inset images show corresponding late gadolinium enhancement imaging demonstrating full thickness late gadolinium enhancement (red arrows).
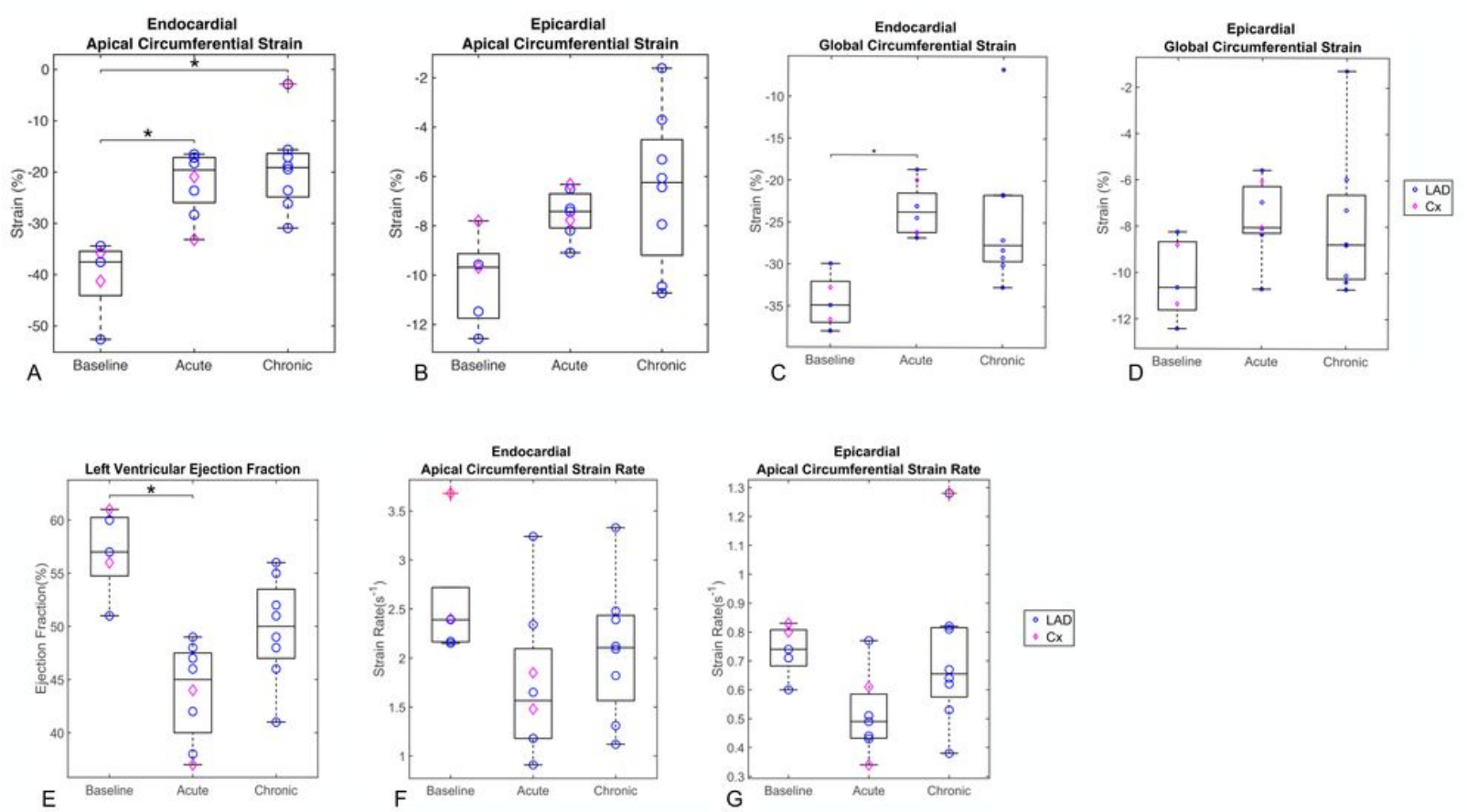

- LAD
$\because \mathrm{Cx}$

\section{Figure 3}


A-D: Overtime changes in apical and global circumferential strains. $A=$ Endocardial apical circumferential strain (ACS); B = Epicardial apical circumferential strain (ACS); C = Endocardial global circumferential strain (GCS); D = Epicardial global circumferential strain (GCS). The two data points at the chronic time point with a red cross (in endocardial ACS and GCS) represent outliers greater than the third quartile plus 1.5 times the interquartile range. E-G: Overtime changes in LVEF, endocardial and epicardial ACS rates. E = Left Ventricular Ejection Fraction (LVEF); F = Endocardial apical circumferential strain (ACS) rates; $G=$ Epicardial ACS rates. The asterisk denotes changes considered significant with $p<0.0024$. Statistical test: Mann-Whitney (* = identifies significant difference)"
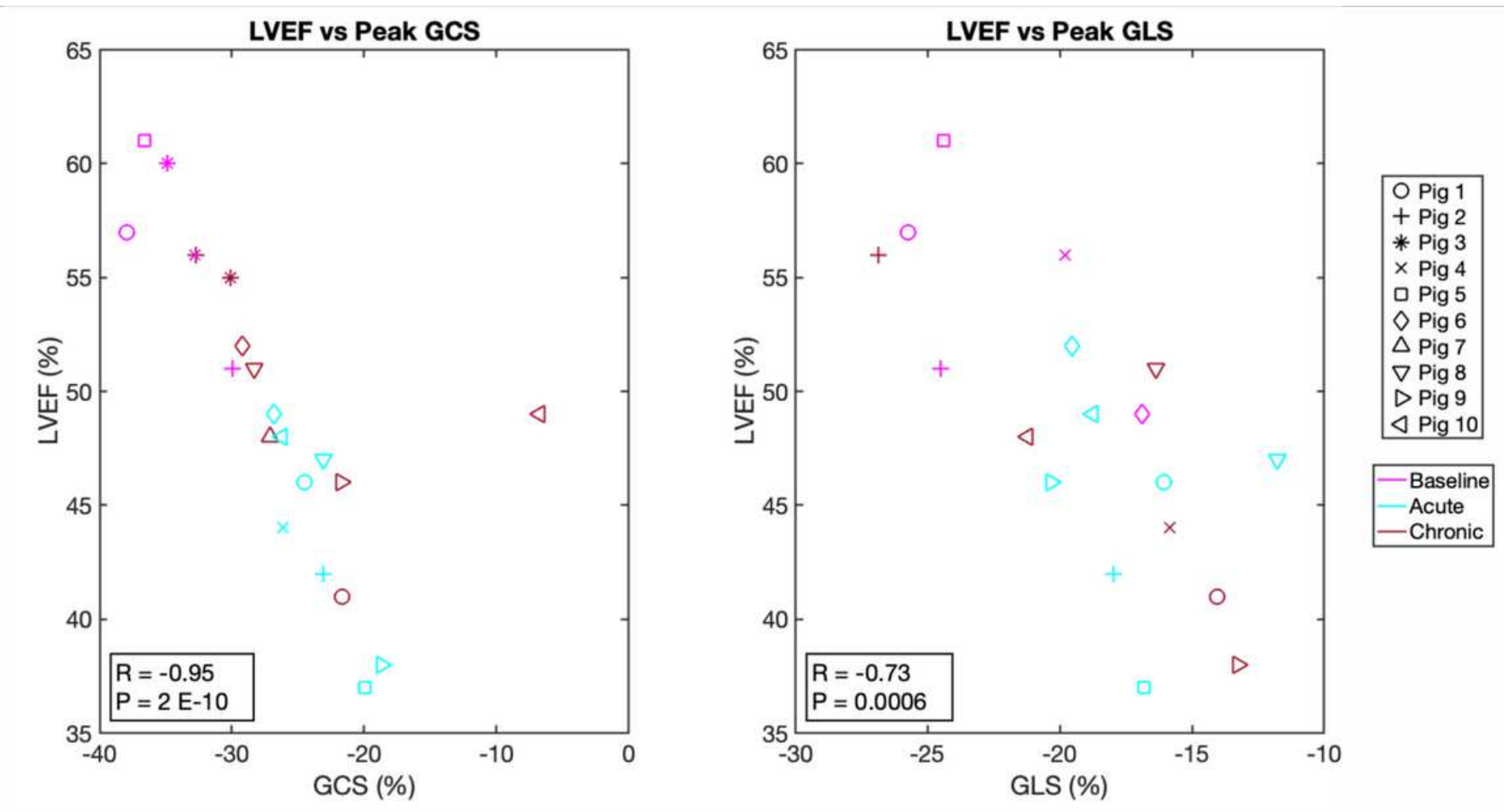

Figure 4

Correlation between endocardial GCS and GLS with LVEF Scatterplot between endocardial GCS and LVEF $(A)$ and between endocardial GLS and LVEF (B). Each individual porcine specimen is denoted by a different marker and three different line colours are used to indicate the experimental time point of baseline, acute, and chronic. 


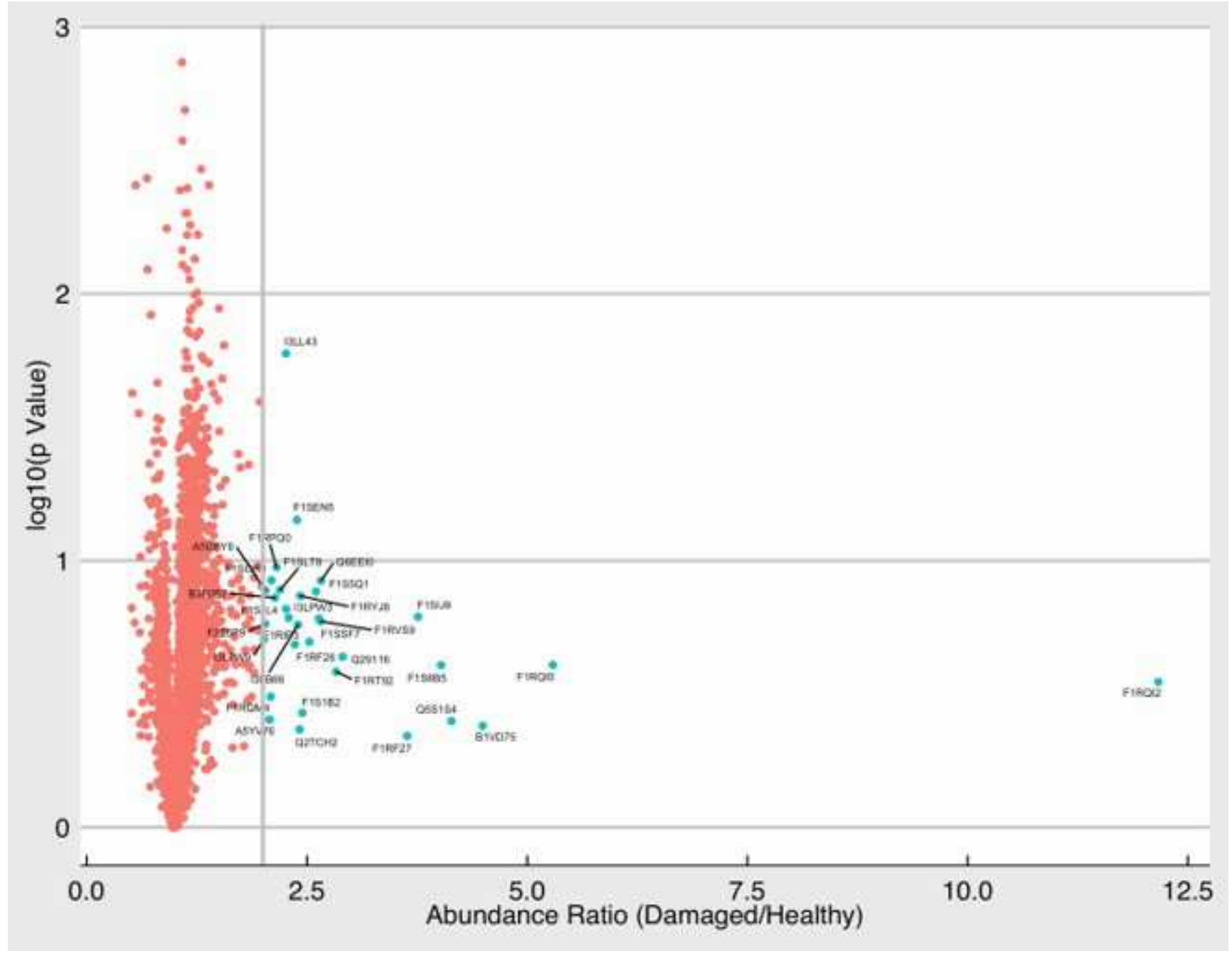

\section{Figure 5}

Volcano plot representation of proteomics Abundance ratios for changes in each protein are shown as $\log 10$ of $p$-value of infarcted/health segments within the same hearts $(n=5)$ 

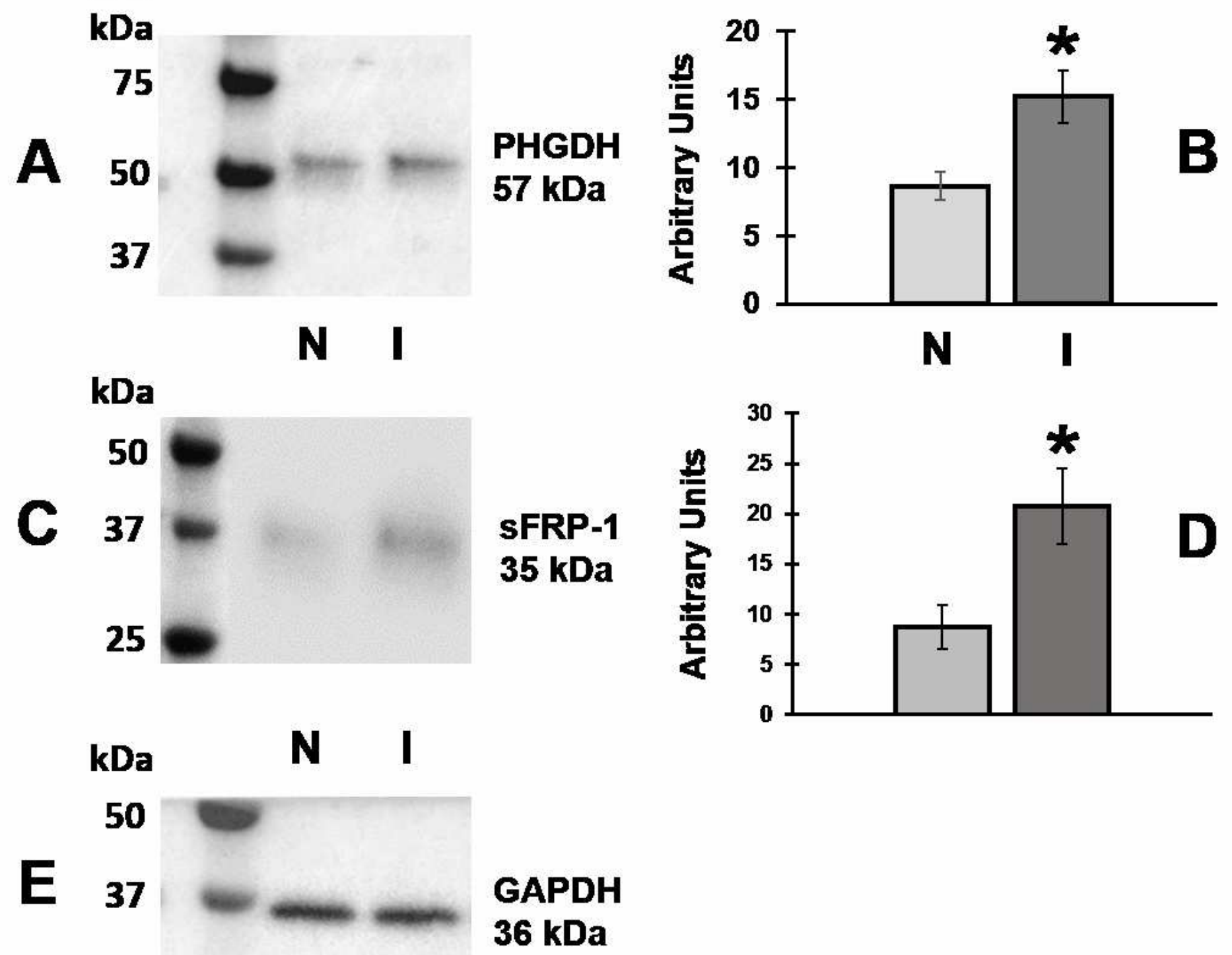

\section{5}

Figure 6

Quantification of D-3PGDH and sFRP1 proteins by western blotting All data presented as Mean \pm SEM; $\mathrm{n}=5$ in each group. Quantification of D-3-phosphoglycerate dehydrogenase (D-3PGDH) and secreted frizzled-related protein 1 (sFRP1) in lysates of the infarcted myocardium (I) and non-infarcted

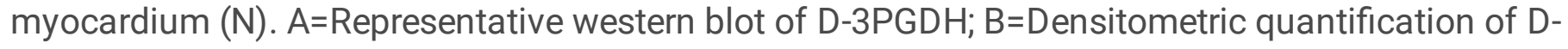
3PGDH; C=Representative western blot for sFRP1; $D=$ Densitometric quantification of sFRP1 expression; $\mathrm{E}=$ Representative western blot for anti-glyceraldehyde 3-phosphate dehydrogenase (GAPDH, used for control of protein loading); ${ }^{*}<0.05$ vs. non-ischaemic myocardium. MW: molecular weight. Statistical Test used: Mann-Whitney. (Figure S1 shows the full blots for all the proteins shown in this figure)

\section{Supplementary Files}


This is a list of supplementary files associated with this preprint. Click to download.

- RevisedSupplementalfile10May2021.docx

- Video1Baselinetimepoint3ch13slice.mov

- Video2Acutetimepoint3ch11slice.mov

- Video3Chronictimepoint3ch10slice.mov 